\title{
Effect of Hoechunyangkyeok-San Extract on Melanogenesis
}

\author{
Mi Jin Kim, Taek Kyu Jung, Hyun-Chul Park, Kyung-Sup Yoon* \\ Skin Science R\&D Center, Saimdang Cosmetics Co., Ltd., Cheongju-si, South Korea \\ Email: "ksyoonjh@hanmail.net
}

Received 14 March 2016; accepted 23 May 2016; published 26 May 2016

Copyright (C) 2016 by authors and Scientific Research Publishing Inc.

This work is licensed under the Creative Commons Attribution International License (CC BY). http://creativecommons.org/licenses/by/4.0/

(c) $\underset{\mathrm{EY}}{\mathrm{i}}$ Open Access

\section{Abstract}

Forsythia fructus has been shown to have antioxidative, anti-inflammatory, antibacterial, antiaging and whitening effects. Hoechunyangkyeok-san (Forsythia viridissima-prescription) is a traditional herbal medicine, which has been clinically used for treating febrile and inflammatory disorders. This work was carried out to investigate the skin whitening effects of Forsythia viridissima-prescription extract (a hydrolyzed extract of Hoechunyangkyeok-san: SID White HYC) on skin. The effects of SID White HYC were assessed the melanin contents in B161 melanoma cells and the pigmented equivalent with HMB45 and Fontana Masson staining in 3D skin model. Then, we examined the expression of major pigment enzymes regulating melanin synthesis and melanosome transport related proteins in B16F1 cells. SID White HYC significantly inhibited the melanin synthesis (56.7\% and $30.6 \%$ inhibition at $100 \mu \mathrm{g} / \mathrm{mL}$, intracellular and secreted, respectively) in B16F1 cells and 3D skin model. In addition, western blotting analysis showed that SID White HYC reduced the expression of melanin synthesis and melanosome transport related proteins in B16F1 cells. In clinical trials, the cream containing $0.05 \%$ SID White HYC showed skin depigmentation effect without any irritation. These results suggest that SID White HYC may be useful inhibition of melanogenesis and melanosome transport. Therefore, SID White HYC may have potential as a skin-whitening ingredient in cosmetics.

\section{Keywords}

Hoechunyangkyeok-San (Formula Extract Containing Forsythia viridissima Fruit, Platycodon grandiflorum Root, Glycyrrhiza glabra Root, Paeonia japonica Root, Angelica gigas Root, Scutellaria baicalensis Root, Gardenia jasminoides Fruit, Coptis chinensis Root, Mentha arvensis Leaf, Rehmannia glutinosa Root, Poncirus trifoliate Fruit), Melanogenesis, Melanosome Transport, Melanin, Whitening

\footnotetext{
${ }^{*}$ Corresponding author.
} 


\section{Introduction}

In mammalian hair and skin, melanogenesis is occurred within melanosomes, the specialized lysosome-related vesicles of melanocytes located in the basal layer of the epidermis [1].

Melanosomes contain three enzymes that are absolutely necessary for the synthesis of both types of melanin (pheomelanin and eumelanin). The first enzyme is tyrosinase, which is responsible for the critical and limiting step in melanogenesis, the hydroxylation of tyrosine to form 3,4-dihydroxyphenylalanine (L-DOPA) followed by oxidation of L-DOPA to form dopaquinone. The second and third enzymes are Tyrosinase-Related Protein-1 (TRP-1) and Dopachrome Tautomerase (DCT), which are more concerned with the generation of different types of melanin [2] [3].

In humans, pigmentation results from the synthesis and distribution of melanin in skin, hair bulbs and eyes [4]. The distribution of melanin in the skin is completed by melanosome transport to keratinocytes. In melanosome transport, there are three kinds of the key player proteins. At the cell dendrite tips, the Rab27a-melanophilin complex recruits the actin-dependent motor protein, myosinVa (Myo5a), allowing melanosomes to move on the actin 4 network. Rab27a, melanophilin (Mlph) and Myo5a make a complex to link melanosomes to the F-actin based motors [5]. Myo5a is a molecular motor that binds to actin filaments through its N-terminal head domain and to the cargo through its C-terminal globular tail [6]. Interaction of Myo5a with melanosomes is mediated by a small Ras-like GTPase Rab27a and Mlph. The GTP bound Rab27a interacts with Mlph that in turn recruits Myo5a to form a tripartite complex [7].

According to a recent study, Rab27a-Mlph and Mlph-Myo5a interactions are very important for melanosome transport. For example, hesperidin blocks the Rab27a-Mlph interaction and therefore causes melanosome aggregation in melanocytes [8]. Also, manassantin B causes melanosome aggregation in melanocytes by blocking the Mlph-Myo5a interaction [9]. From these studies, we know that melanosome transport is influenced by inhibiting interaction of tripartite complex as well as loss of any one of the components of the tripartite complex.

Hoechunyangkyeok-san is a traditional herbal prescription for treating febrile and inflammatory diseases, which recorded in Manbyeonghoechun (which means "Restoration of all illnesses”, 1587 at first) and Bangyakhappyeon, clinical herbal prescription book [10] [11]. It consists of Forsythia viridissima fruit, Platycodon grandiflorum root, Glycyrrhiza glabra root, Paeonia japonica root, Angelica gigas root, Scutellaria baicalensis root, Gardenia jasminoides fruit, Coptis chinensis root, Mentha arvensis leaf, Rehmannia glutinosa root, and Poncirus trifoliata fruit. Hoechunyangkyeok-san has been reported to be a useful treatment for diabetes, atherosclerosis and hyperlipidemia in the type 1 diabetic model, and inhibit melanin content in B16F1 cells [12] [13]. However, it has been rarely conducted to evaluate the skin whitening activities. To search for new skin-lightening agents, we investigated the effect of Hoechunyangkyeok-san on depigmenting mechanisms.

\section{Materials and Methods}

\subsection{Chemicals and Reagents}

$\alpha$-MSH, 3-(4,5-dimetylyhiazol-2-yl)-2,5-diphenyltetrazoliumbromide (MTT) and dimethylsulfoxide (DMSO) were purchased from Sigma-Aldrich (USA). Penicillin/streptomycin, phosphate buffered saline (PBS), fetal bovine serum (FBS) and tryps in were purchased from Gibco (USA). Dulbecco's modified Eagle's medium (DMEM) was obtained from HyClone (USA). Antibodies recognizing tyrosinase, TRP-1, -2, MITF and Rab27a were purchased from Santa Cruz Biotechnology (USA), $\beta$-actin and Myo5a were purchased from Cell Signaling Technology (USA). Anti-Mlph was bought from Proteintech (USA).

\subsection{Preparation of SID White HYC}

Herbs which constitute Hoechunyangkyeok-san were purchased from a Korean herbal medicine dealer in Gumediherb, Korea and composed of 11 herbs shown as Table 1 [11].

Hoechunyangkyeok-san (50 g, in the ratio of Table 1) was extracted twice with $75 \%$ aqueous ethanol under reflux at $60^{\circ} \mathrm{C}-90^{\circ} \mathrm{C}$ for 4 hours, filtered, and evaporated under reduced pressure. The 75\% aqueous ethanol extract was redissolved in $10 \%$ aqueous ethanol and hydrolyzed by enzymes, Pectinex (Novozyme, Denmark) at $50^{\circ} \mathrm{C}-60^{\circ} \mathrm{C}$ for 30 hours and fractionated by ethyl acetate. The fraction was cooled, filtered, and evaporated under reduced pressure. Finally we obtained the SID White HYC. 
Table 1. The composition of Hoechunyangkyeok-san.

\begin{tabular}{cc}
\hline Herbal name & Amount (\%) \\
Forsythia viridissima fruit & 14.70 \\
Platycodon grandiflorum root & 8.53 \\
Glycyrrhiza glabra root & 8.53 \\
Paeonia japonica root & 8.53 \\
Angelica gigas root & 8.53 \\
Scutellaria baicalensis root & 8.53 \\
Gardenia jasminoides fruit & 8.53 \\
Coptis chinensis root & 8.53 \\
Mentha arvensis leaf & 8.53 \\
Rehmannia glutinosa root & 8.53 \\
Poncirus trifoliata fruit & 8.53 \\
\hline
\end{tabular}

\subsection{Cell Culture}

B16F1 murine melanoma cells were obtained from the American Type Culture Collection (ATCC, USA) and cultured in DMEM supplemented with penicillin $(100 \mathrm{U} / \mathrm{mL})$, streptomycin $(100 \mu \mathrm{g} / \mathrm{mL})$ and $10 \%$ FBS. Cells were incubated at $37^{\circ} \mathrm{C}$ in a humidified atmosphere containing $5 \% \mathrm{CO}_{2}$.

\subsection{Cytotoxicity (MTT) Assay}

Cytotoxicity was evaluated using the MTT assay. The B16F1 cells were seeded at a density of $5 \times 10^{3}$ cells/well in a 96-well plate, and were cultured at $37^{\circ} \mathrm{C}$ with $5 \% \mathrm{CO}_{2}$. The cells were then treated with SID White $\mathrm{HYC}$ at various concentrations $(1,20,50,100 \mu \mathrm{g} / \mathrm{mL})$ for 72 hours. Following incubation, the cells were treated with the MTT solution for 4 hours at $37^{\circ} \mathrm{C}$. The supernatants were aspirated, and DMSO was added to each well. After incubation for 20 minutes, the absorbance was measured at $540 \mathrm{~nm}$ using a microplate reader (Synergy, BioTek, USA). Cytotoxicity is presented as a percentage of the optical density of the vehicle group.

\subsection{Measurement of Intracellular and Secreted Melanin Contents}

The intracellular and secreted melanin content was measured using a slight modification of a previously reported method [14] [15]. Briefly, B16F1 cells were incubated at a density of $5 \times 10^{4}$ cells/well in a 6-well plate overnight. B16F1 cells, pretreated with the indicated concentrations of SID White HYC for 1 hour, were treated for 72 hours with $\alpha$-MSH $(200 \mathrm{nM})$. In order to measure the secreted melanin, $200 \mu \mathrm{L}$ aliquots of media were placed in 96-well plates and absorbance was measured at $405 \mathrm{~nm}$ using a microplate reader. Cells were detached by incubation in trypsin/EDTA. After precipitation, the color of the cell pellets was evaluated visually, and pellets were solubilized in $1 \mathrm{~N} \mathrm{NaOH}$ containing 10\% DMSO. Spectrophotometric analysis of cellular melanin content was performed by quantifying absorbance at $405 \mathrm{~nm}$. Three independent experiments were performed in triplicate.

\subsection{Cellular Tyrosinase Assay}

Tyrosinase activity in B16F1 cells was assayed as described previously with modifications [16] [17]. Cells were washed twice with ice-cold phosphate buffered saline (PBS) and lysed with cell lysis buffer (0.1\% Triton-X 100 in $0.1 \mathrm{M}$ phosphate buffer, $\mathrm{pH}$ 6.8). The reaction mixture containing $40 \mu \mathrm{L}$ of $10 \mathrm{mM}$ L-tyrosine, $140 \mu \mathrm{L}$ of enzyme solution (lysate $40 \mu \mathrm{g}$ in $0.1 \mathrm{M}$ phosphate buffer, $\mathrm{pH} 6.8$ ) and $20 \mu \mathrm{L}$ of indicated concentration SID White $\mathrm{HYC}$ (in PBS) was incubated at $37^{\circ} \mathrm{C}$ for 10 minutes. The absorbance of samples was measured at $475 \mathrm{~nm}$. Enzyme activities were expressed as a percentage of vehicle. 


\subsection{Western Blot Analysis}

Cells were rinsed twice with PBS and scraped into lysis buffer. Lysates were prepared with lysis buffer according to the manufacturer's instructions (Thermo, USA; iNtRON, Korea). The protein concentration was determined using a BCA Assay Reagent Kit (Thermo Fisher Scientific Inc., USA). Proteins present in the cell lysates were separated by Sodium Dodecyl Sulfate-Polyacrylamide Gel Electrophoresis (SDS-PAGE) using a 4\% - 20\% gel, transferred onto polyvinylidene fluoride membranes (GE Healthcare, UK), blocked with 5\% skim milk, and treated with primary antibodies for 2 hoursat room temperature. After incubation with horseradish peroxidaseconjugated secondary antibodies (Santa Cruz Biotechnology, USA) at room temperature, immunoreactive proteins were detected with a chemiluminescent ECL assay kit (GE Healthcare, UK) according to the manufacturer's instructions. Bands were visualized using a ChemiDoc image analyzer (BIO-RAD, USA).

\subsection{Pigmented Reconstructed Skin Model}

Reconstructed human epidermis MelaSkin ${ }^{\mathrm{TM}}$ (MCTT, Korea) consisted of normal human derived epidermal keratinocytes and melanocytes that had been cultured to form a multilayered, highly differentiated model. Reconstructed epidermis was placed in 6-well plates and incubated at $37^{\circ} \mathrm{C}$ in a humidified atmosphere of $5 \% \mathrm{CO}_{2}$ in air. The tissue was incubated for 24 hours with the indicated concentration of SID White HYC.

\subsection{Histology and Immunohistochemistry}

After 24 hours, tissue were fixed in 10\% formaldehyde for 1 day and processed for conventional paraffin embedment. $4 \mu \mathrm{m}$ thickness sections were then prepared. Sections were stained with hematoxylin and eosin (H\&E), HMB-45 (Santa Cruz Biotechnology, USA) and Fontana-Masson for light microscopic observation.

\subsection{Clinical Evaluation}

A randomized, double-blind, placebo-test (0.05\% SID White HYC containing cream), split-face comparison study was conducted. All the participants provided informed written consent prior to their participation. The participants were twenty-two healthy female volunteers aged 37 - 54 years old (average $48.05 \pm 4.40$ years).

\subsubsection{Clinical Assessment}

Visual assessment was done at before, 4 and 8 weeks after treatment. Two investigator clinically graded skin color base on 10 points scale at each visit ( 0 point, clear and transparent; 10 point, dark and unclear). The mean value of the data obtained from each investigator was then calculated and compared.

\subsubsection{Objective Evaluation}

Objective measurement was done at before, 4 and 8 weeks after treatment. Objective skin color measurements were performed using a Mexameter (MX-18, Courage \& Khazaka Electronic GmbH, Cologne, Germany) and Spectrophotometer (CM-2500d, Minolta, Japan) during each visit. Three successive measurements of the Mexameter and Spectrophotometer were made on the darkest portion of the test and placebo cream treated skin. The mean value of the data obtained from each exam was then calculated and compared. Melanin index was measured with Mexameter. High melanin index score indicates dark skin color. The $L, a$ and $b$ values were recorded with Spectrophotometer. ( $L^{*}$ : Luminance parameters (brightness), $a^{*}$ : Chrominance parameters (green-to-red), $b^{*}$ : Chrominance parameters (blue-to-yellow)). All the experimental data were expressed as means \pm SEM. Individual typology angle $\left(\mathrm{ITA}^{\circ}\right)$ value was achieved by the formulation. Low ITA ${ }^{\circ}$ value indicates dark skin color.

$$
\text { ITAp value }=\left[\text { Arc Tangent }\left\{\left(L^{*}-50\right) / b^{*}\right\}\right] \times 180 / 3.14159
$$

\subsubsection{Statistical Analysis}

For statistical analysis, an RM ANOVA method was used to compare the change in the visual assessment score, melanin index score and $\mathrm{ITA}^{\circ}$ value resulting from treatment between test and placebo treated skin. The data was analyzed using SPSS Package Program 11.5 (IBM, USA). $P$-values $<0.05$ were considered statistically significant. 


\subsection{Statistical Analysis}

Data were analyzed using Sigma Plot. Results were expressed as the mean \pm S.D. of three independent experiments. Comparisons were performed using a one-way ANOVA followed by Duncan's multiple range test. A $P$-value $<0.05$ was considered statistically significant.

\section{Results}

\subsection{Effect of SID White HYC on Cell Viability and Melanin Biosynthesis}

B16F1 cells were exposed to various concentration of SID White HYC, and cell viability was measured by MTT assay. As shown in Figure 1, cell viability of SID White HYC was above $80 \%$ up to $100 \mu \mathrm{g} / \mathrm{mL}$ (Figure 1).

To investigate the inhibitory effect of SID White HYC on melanogenesis, the melanin contents of the B16F1 cells were measured after treatment with SID White HYC. Cells were exposed to SID White HYC $(10,50,100$ $\mu \mathrm{g} / \mathrm{mL})$ for 72 hours with $\alpha$-MSH $(200 \mathrm{nM})$, and then the amounts of melanin intracellular spaces and secreted into the medium were measured individually. As Figure 2 shows, SID White HYC decreased the amount of melanin intracellular spaces and secreted into the media (at $100 \mu \mathrm{g} / \mathrm{mL}, 56.72 \%$ and $30.64 \%$ inhibition, respectively). These results indicate that SID White HYC might have inhibited the synthesis of melanin.

\subsection{Inhibitory Effect of SID White HYC on Tyrosinase Activity}

SID White HYC inhibited cellular tyrosinase activity in a dose dependent manner but the effect did not inhibit a mushroom tyrosinase activity (data not shown). SID White HYC at $50 \mu \mathrm{g} / \mathrm{mL}$ reduced tyrosinase activity by $35 \%$ (Figure 3).

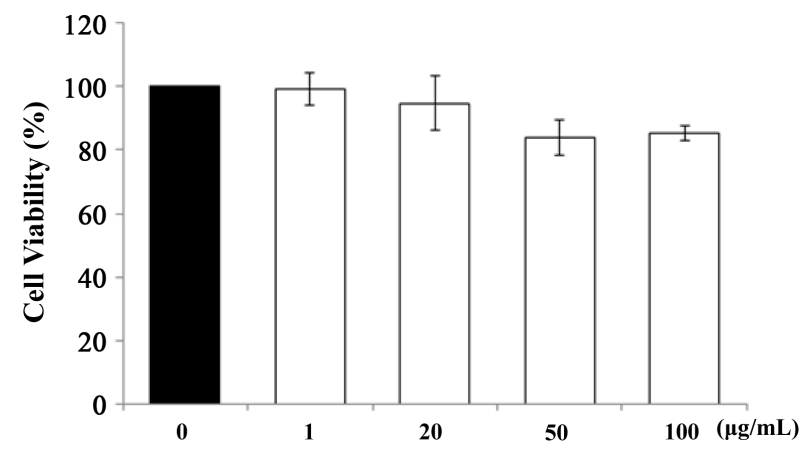

Figure 1. Cell viability of SID White HYC in B16F1 cells. The cells were treated with various concentration of samples for 72 hours. Data are the mean \pm S.D from three independent experiments. ${ }^{*} P<0.05$ : statistically significant vs. control group (vehicle).

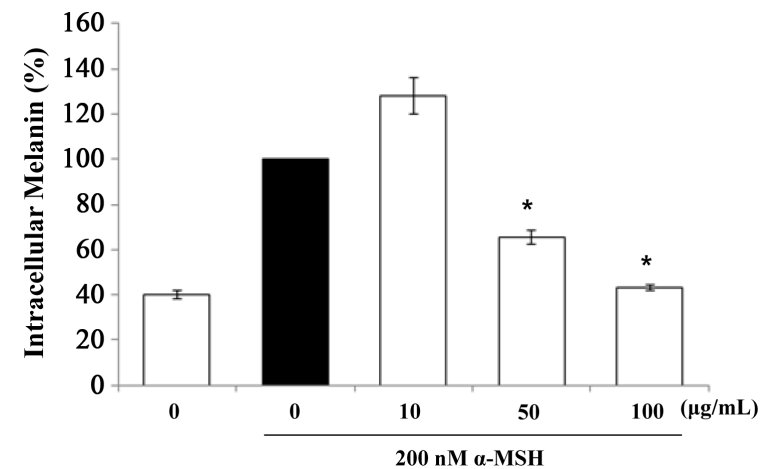

(a)

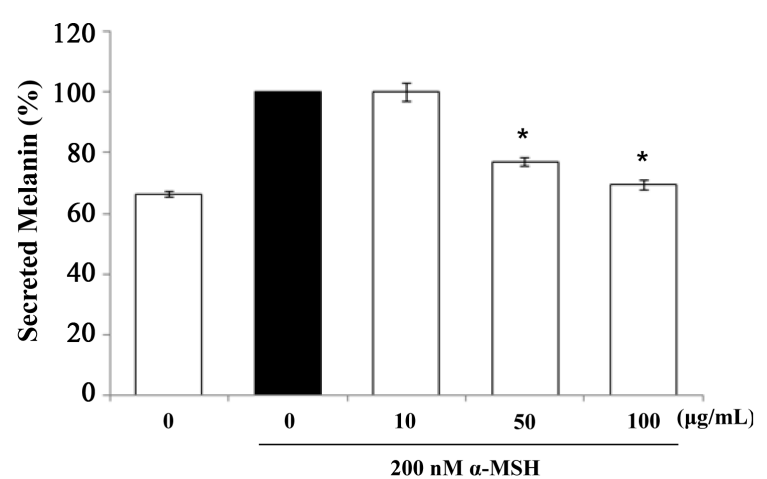

(b)

Figure 2. The effect of SID White HYC on intracellular (a) and secreted melanin (b) inmurine B16F1 melanoma cells treated with $\alpha$-MSH. Thecells were treated with $\alpha$-MSH and SID White HYC at indicated concentration for 72 hours. Data are the mean \pm S.D from three independent experiments. ${ }^{*} P<0.05$ vs. control group (vehicle). 


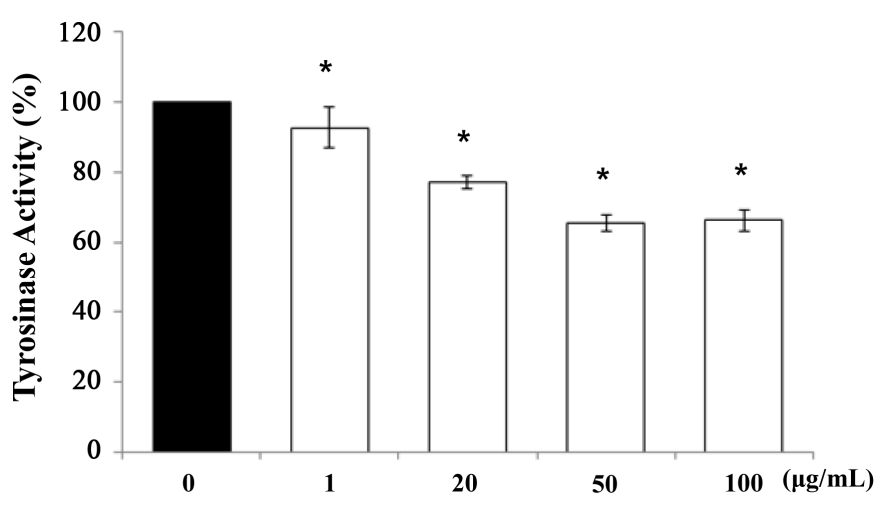

Figure 3. The effect of SID White HYC on cellular tyrosinase activity. Data are the mean \pm S.D from three independent experiments. ${ }^{*} P<0.05$ vs. control group (vehicle).

\subsection{Inhibitory Effect of SID White HYC on Melanogenesis-Related Proteins Expression}

The expression levels of melanogenesis-related proteins were determined by western blot in $\alpha$-MSH stimulated B16F1 cells. Protein levels of tyrosinase and TRP-1 were significantly decreased but not TRP-2 after treatment with SID White HYC (Figure 4). These results suggest that SID White HYC decease melanin synthesis by down-regulating of melanogenesis-related proteins.

\subsection{Inhibitory Effect of SID White HYC on Melanosome Transport-Related Proteins Expression}

The function of melanosomes in skin pigmentation requires not only melaninformation, but also transport within the cell. Melanosomes are transported along microtubules towards actin near the plasma membrane and in the tips of dendrites, from where they are transferred to keratinocytes [18]. In melanocytes, the transport of melanosome is carried out by a complex of three proteins: Rab27a, Mlph and Myo5a.

To know the effects of SID White HYC on triplicate protein involved in melanosome transport, we checked expression level of Rab27a, Mlph and Myo5a after treatment with SID White HYC for 72 hours on B16F1 cells. There was dose-dependent reduction of Rab27a, Mlph and Myo5a expression with SID White HYC treatment compared with control (Figure 5). These results demonstrate that SID White HYC decease melanin secretion by down-regulating of melanosome transport-related proteins.

\subsection{Effect of SID White HYC on Melanogenesis in Cultured Human Skin Model}

To identify the effects of SID White HYC on skin pigmentation, we used three-dimensional cultured human skin model (MelaSkin ${ }^{\mathrm{TM}}$ ). The MelaSkin exhibits in vivo-like morphological and ultrastructural characteristics. When MelaSkin was cultured in media, melanogenesis was induced naturally. Thus the model provides a useful in vitro means to evaluate cosmetic and pharmaceutical agents designed to modulate skin pigmentation. Different concentration of SID White HYC and the vehicle were applied on the reconstructed skin for 24 hours.

Distribution of melanin in MelaSkin was shown by Fontana-Masson and HMB45 staining. Melanin is stained strongly by Fontana-Masson stain in all layers of the epidermis. One of the most frequently used melanocytic markers in clinical practice is HMB45. HMB-45 is a monoclonal antibody that reacts against an antigen present in melanocytic tumors such as melanomas but not other tumors, thus demonstrating specificity and sensitivity [19].

Figure 6 showed decreased deposition of pigment granule in MelaSkin equivalents following SID White HYC treatment. Fontana-Masson and HMB45 staining revealed the hypopigmentation activities of SID White HYC.

\subsection{Clinical Evaluation}

Figure 7 displays the decrease of the clinical visual assessment score over time compared with before. A decrease in the visual assessment score over time was observed for both test and placebo treated skin. The visual assessment score of the test group was significantly lower than that of the placebo group $(P<0.05)$. The visual 


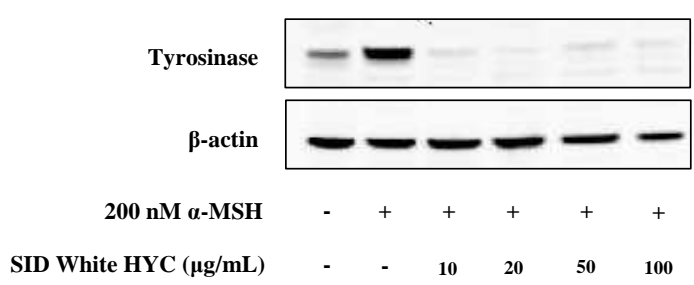

(a)

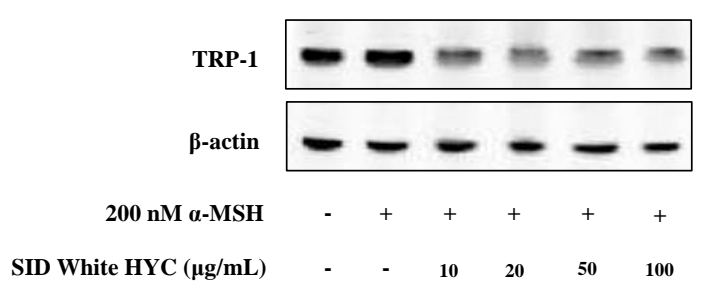

(b)

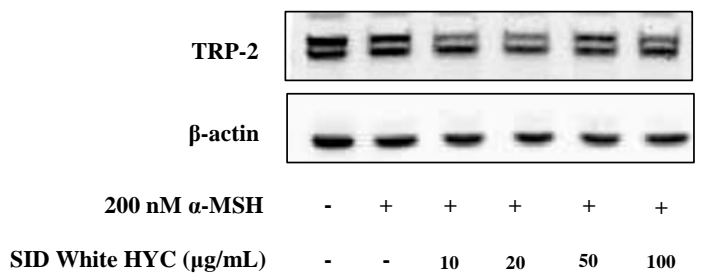

(c)

Figure 4. Expression of melanogenesis-related proteins in B16F1 cells treated with SID White HYC. The expression levels of tyrosinase (a); TRP-1 (b) and TRP-2 (c) were examined by western blot.

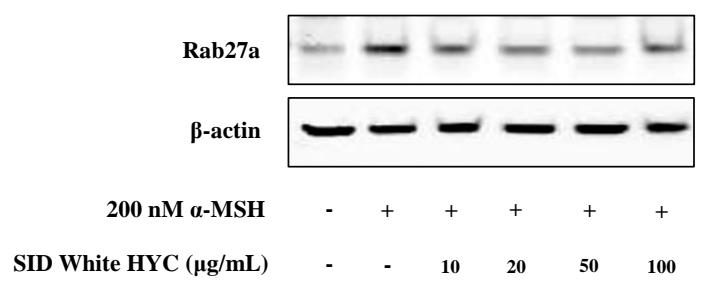

(a)

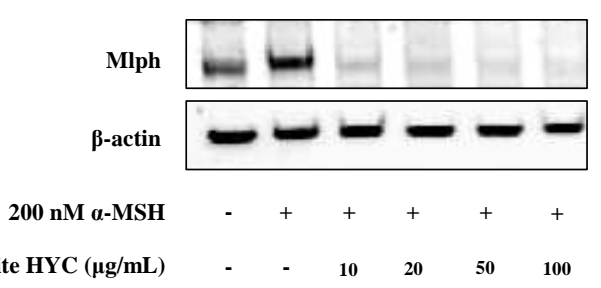

(b)

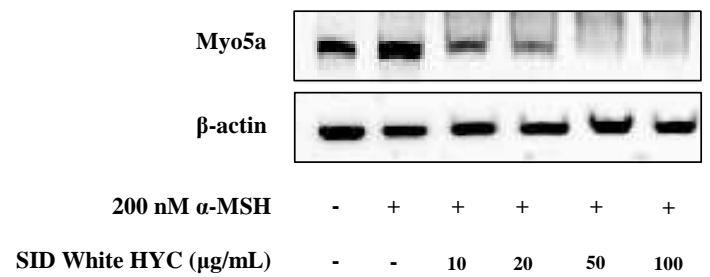

(c)

Figure 5. Expression of melanosome transport-related proteins in B16F1 cells treated with SID White HYC. The expression levels of Rab27a (a); Mlph (b) and Myo5a (c) were examined by western blot. 
assessment score change at the end of the study for the test and placebo treated skin were $-2.5 \%$ and $-0.43 \%$ respectively, with a significant different after 8 weeks $(P<0.05)$ (Figure 7(a)).

A significant decrease in the melanin index score was observed from 4 week through the end of study at both group (Figure 7(b)). The mean melanin index score change at the end of the study for the test and placebo treated skin were $-5.08 \%$ and $-2.48 \%$ respectively, with a statistically significant difference $(P<0.05)$.

At the Spectrophotometer analysis, the test group also showed significant increase in ITA ${ }^{\circ}$ value after 4 week of treatment, which lasted until the end of the study. The $\mathrm{ITA}^{\circ}$ value change at the end of the study for the test and the placebo treated skin were $+11.72 \%$ and $+7.82 \%$ respectively, with a statistically significant difference ( $P$ $<0.05$ ) (Figure 7(c)).

\section{Discussion}

Depigmentation can be accomplished by specific interaction of an active: before melanin synthesis; during melanin synthesis; after melanin synthesis [20].

Skin pigmentation results from melanin synthesis by melanocytes, which is induced by exposure to UV radiation. In mammals, melanin synthesis is stimulated by a large number of effectors, including UV-B radiation and cAMP-elevating agents (forskolin, 3-isobutyl-1-methylxanthine, $\alpha$-melanocytestimulating hormone ( $\alpha$-MSH), glycyrrhizin) [21]-[25].

Melanin synthesis takes place within specialized intracellular organelles named melanosome, where the enzymes involved in melanogenesis, including tyrosinase, are located. Mature melanin-filled melanosomes move from the perinuclear region to the dendrite tips of melanocytes and are transferred to neighboring keratinocytes by a mechanism that is still now well characterized. The result of this process is a uniform distribution of melanin pigments in the epidermis that is responsible for skin and hair color in humans and other animals. The ability to inhibit melanin synthesis effectively is very important in regulating pigmentation and skin whitening [26] [27].

With regard to the inhibitory effect of SID White HYC on melanogenesis, SID White HYC reduced the synthesis of melanin in $\alpha$-MSH stimulated B16F1 cells (Figure 2). According to the HMB45 immunostaining assay, in which HMB45 was found to react with the neuraminidase-sensitive sialylated glycol-conjugate present in early-stage melanosomes, the decreased presence of HMB45 indicated that SID White HYC inhibited the formation of melanosome (Figure 6). The results of Fontana-Masson staining were in complete agreement with this observation, confirming that SID White HYC influenced active melanosome formation and thus melanocytic differentiation. SID White HYC reduced the activity of cellular tyrosinase but the effect was not a direct inhibition of tyrosinase, since it had no effect on mushroom tyrosinase (Figure 3). These data suggest that SID White HYC has an influence on the expression of melanogenic proteins such as tyrosinase, TRP-1 and TRP-2. The changes in the level of melanogenic proteins expression analyzed by immunoblot indicate that SID White HYC decreased melanogenic proteins level (Figure 4).

In melanosome transport, there are three kinds of the key player proteins. Rab27a, Mlph and Myo5a form a tripartite complex that allows interaction of melanosomes with the actin network [28] [29]. When the three constituents meet each other to form a complex, it is possible to normally transport melanosome to the periphery. If any of the three constituents is abnormal, it is observed that a problem arises in the transport of melanosomes without causing any problem in the production of melanin, so that the color of the skin becomes white.

In regard to the inhibitory effect of SID White HYC on melanogenesis, SID White HYC reduced the secreted melanin in $\alpha$-MSH stimulated B16F1 cells (Figure 2(b)). It seems that SID White HYC has an influence on melanosome transport. The changes in the level of transport-related proteins expression analyzed by immunoblot indicate that SID White HYC decreased transport-related proteins level (Figure 5). These results has led to development of effective skin-whitening ingredients with blocking the before and after melanin synthesis.

The levels of skin pigmentation of the test and placebo groups were measured at 4 and 8 weeks using the Mexameter and Spectrophotometer, melanin index and ITA ${ }^{\circ}$, respectively. The test group had significantly less pigmentation throughout the study compared with before (Figure 7). The ITA $^{\circ}$ value is believed to provide a quantitative means for classifying individual skin color [30]. It indicates the melanotic status of the skin at the time of the testing to be taken into account [31] [32]. None of adverse reactions such as erythema, burning sensation and pruritus were observed during 8 weeks of study period. These results suggest that SID White HYC ameliorates darkness of the skin. 


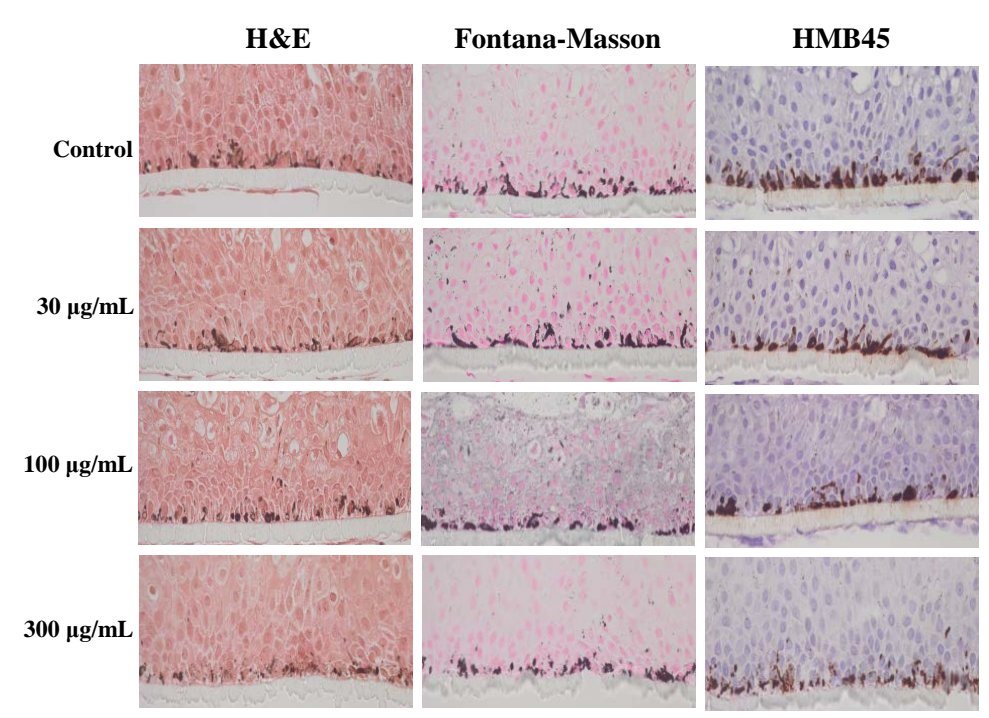

Figure 6. Histological analysis of melanin deposition in cultured human skin model treated with SID White HYC using Fontana-Masson and HMB 45 stains.

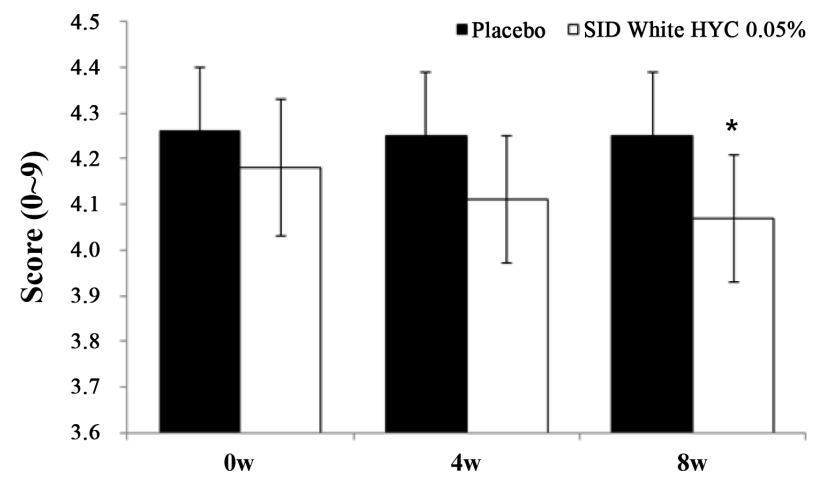

(a)

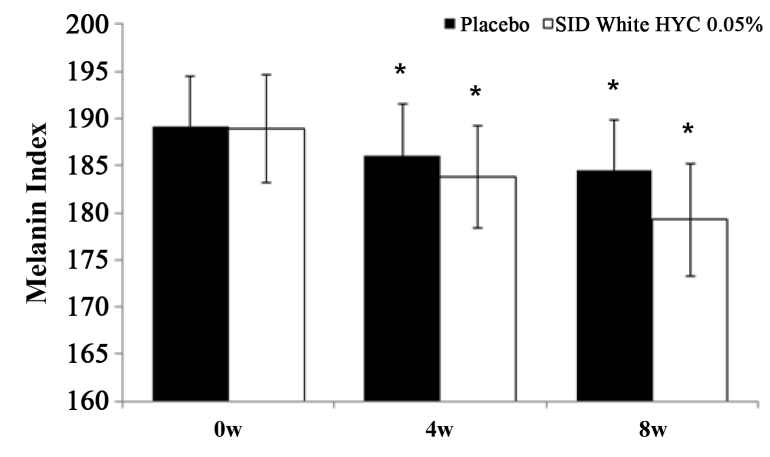

(b)

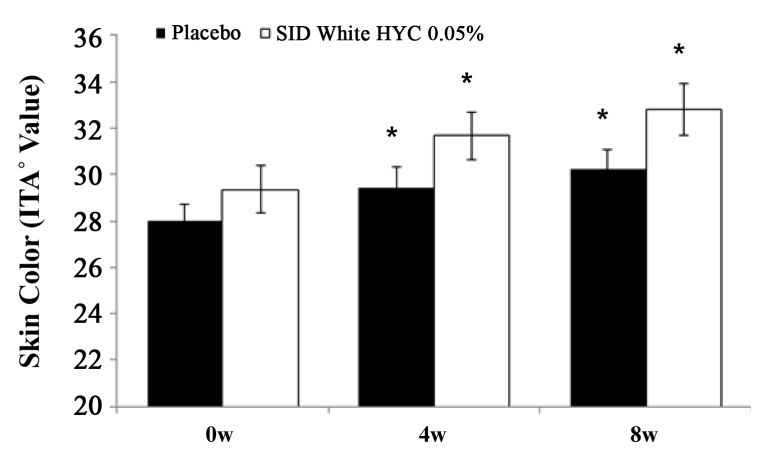

(c)

Figure 7. The effects of SID White HYC containing cream on the visual assessment score (a); melanin index (b) and ITA ${ }^{\circ}$ value (c) compared with placebo. Mean \pm SEM, ${ }^{*} P<0.05$ vs. before treatment.

\section{Conclusion}

Taken together, SID White HYC could be expected to exert skin whitening effect by decrease of the melanogenesis-related proteins and melanosome transport-related proteins expression without any adverse effects. In conclusion, these studies demonstrate that SID White HYC may be useful inhibitor of melanogeneiss and that SID White HYC may be an effective component in cosmetics designed to lighten skin. 


\section{Acknowledgements}

This work was supported by Chungcheong Institute for Regional Program Evaluation Promotion Project (R0002893) of the Ministry of Trade, Industry and Energy Republic of Korea.

\section{References}

[1] Matesic, L.E., Yip, R., Reuss, A.E., Swing, D.A., O’Sullivan, T.N., Fletcher, C.F., Copeland, N.G. and Jenkins, N.A. (2001) Mutations in Mlph, Encoding a Member of the Rab Effector Family, Cause the Melanosome Transport Defects Observed in Leaden Mice. Proceedings of the National Academy of Sciences of the United States of America, 98, 10238-10243. http://dx.doi.org/10.1073/pnas.181336698

[2] Hearing, V.J. and Yamaguchi, Y. (2006) Melanocyte Distribution and Function in Human Skin: Effects of Ultraviolet Tradiation. In: Hearing, V.J. and Leong, S.P.L., Eds., From Melanocytes to Melanoma: The Progression to Malignancy, Humana Press, New Jersey, 101-115. http://dx.doi.org/10.1007/978-1-59259-994-3

[3] Slominski, A., Tobin, D.J., Shibahara, S. and Wortsman, J. (2004) Melanin Pigmentation in Mammalian Skin and Its Hormonal Regulation. Physiological Reviews, 84, 1155-1228. http://dx.doi.org/10.1152/physrev.00044.2003

[4] Goding, C.R. (2007) Melanocytes: The New Black. The International Journal of Biochemistry \& Cell Biology, 39, 275-279. http://dx.doi.org/10.1016/j.biocel.2006.10.003

[5] Desnos, C., Huet, S. and Darchen, F. (2007) “Should I Stay or Should I Go?”: Myosin V Function in Organelle Trafficking. Biology of the Cell, 99, 411-423. http://dx.doi.org/10.1042/BC20070021

[6] Bahadoran, P., Busca, R., Chiaverini, C., Westbroek, W., Lambert, J., Bille, K., Valony, G., Fukuda, M., Naeyaert, J.M., Ortonne, J.P. and Ballotti, R. (2003) Characterization of the Molecular Defects in Rab27a, Caused by RAB27A Missense Mutations Found in Patients with Griscelli Syndrome. The Journal of Biological Chemistry, 278, 1138611392. http://dx.doi.org/10.1074/jbc.M211996200

[7] Fukuda, M. and Itoh, T. (2004) Slac2-a/Melanophilin Contains Multiple PEST-Like Sequences That Are Highly Sensitive Toproteolysis. The Journal of Biological Chemistry, 279, 22314-22321. http://dx.doi.org/10.1074/jbc.M401791200

[8] Kim, B., Lee, J.Y., Lee, H.Y., Nam, K.Y., Park, J., Lee, S.M., Kim, J.E., Lee, J.D. and Hwang, J.S. (2013) Hesperidin Suppresses Melanosome Transport by Blocking the Interaction of Rab27A-Melanophilin. Biomolecules \& Therapeutics, 21, 343-348. http://dx.doi.org/10.4062/biomolther.2013.032

[9] Chang, H., Choi, H., Joo, K.M., Kim, D. and Lee, T.R. (2012) Manassantin B Inhibits Melanosome Transport in Melanocytes by Disrupting the Melanophilin-Myosin Va Interaction. Pigment Cell \& Melanoma Research, 25, 765-772. http://dx.doi.org/10.1111/pcmr.12002

[10] Gong, J.H. (1972) Man-byeong-hoe-chun. Haeng-rim-seo-won, Seoul, 16.

[11] Hwang, D.Y. (1985) Taeyok Chumgmaek Bangyakhappyeon. Namsandang Editorial Bureau, Seoul, 229-230.

[12] Hong, J.S. (1991) Effect of Hoechounyanggeuksan on the Glucose in Diabetic Rats Induced by Streptozotocin. The Kyung Hee University Oriental Medical Journal, 14, 397-411.

[13] Kim, M.J., Jung, T.K. and Yoon, K.-S. (2015) Anti-Aging Effects of Prescription Extracts Containing Forsythia viridissima L. Journal of the Society of Cosmetic Scientists of Korea, 41, 85-96. http://dx.doi.org/10.15230/SCSK.2015.41.1.85

[14] Oka, M., Ichihashi, M. and Chakraborty, A.K. (1996) Enhanced Expression of Protein Kinase C Subspecies in Melanogenic Compartments in B16 Melanoma Cells by UVB or MSH. The Journal of Investigative Dermatology, 106, 377378. http://dx.doi.org/10.1111/1523-1747.ep12343168

[15] Siegrist, W. and Eberle, A.N. (1986) In Situ Melanin Assay for MSH Using Mouse B16 Melanoma Cells in Culture. Analytical Biochemstry, 159, 191-197. http://dx.doi.org/10.1016/0003-2697(86)90327-1

[16] Choi, Y.M., Jun, H.J., Dawson, K., Rodriguez, R.L., Roh, M.R., Jun, J., Choi, C.H., Shim, J.H., Lee, C.H., Lee, S.J., Park, K.H., et al. (2010) Effects of the Isoflavone puerarin and Its Glycosides on Melanogenesis in B16 Melanocytes. European Food Research and Technology, 231, 75-83. http://dx.doi.org/10.1007/s00217-010-1251-5

[17] Lerch, K. (1987) Monophenol Monooxygenase from Neurospora crassa. Methods in Enzymology, 142, 165-169. http://dx.doi.org/10.1016/S0076-6879(87)42025-9

[18] Scott, G. (2002) Rac and Rho: The Story behind Melanocyte Dendrite Formation. Pigment Cell Research, 15, 322-330. http://dx.doi.org/10.1034/j.1600-0749.2002.02056.x

[19] Gown, A.M., Vogel, A.M., Hoak, D., Gough, F. and McNutt, M.A. (1986) Monoclonal Antibodies Specific for Melanocytic Tumors Distinguish Subpopulations of Melanocytes. The American Journal of Pathology, 123, 195-203.

[20] Briqanti, S., Camera, E. and Picardo, M. (2003) Chemical and Instrumental Approaches to Treat Hyperpigmentation. 
Pigment Cell Research, 16, 101-110. http://dx.doi.org/10.1034/j.1600-0749.2003.00029.x

[21] Friedmann, P.S. and Gilchrest, B.A. (1987) Ultraviolet Radiation Directly Induces Pigment Production by Cultured Human Melanocytes. Journal of Cellular Physiology, 133, 88-94. http://dx.doi.org/10.1002/jcp.1041330111

[22] Wong, G. and Pawelek, J. (1975) Melanocyte-Stimulating Hormone Promotes Activation of Pre-Existing Tyrosinase Molecules in Cloudman S91 Melanoma Cells. Nature, 255, 644-646. http://dx.doi.org/10.1038/255644a0

[23] Halaban, R., Pomerantz, S.H., Marshall, S. and Lerner, A.B. (1984) Tyrosinase Activity and Abundance in Cloudman Melanoma Cells. Archives of Biochemistry and Biophysics, 230, 383-387. http://dx.doi.org/10.1016/0003-9861(84)90121-8

[24] Hunt, G., Todd, C., Creswell, J.E. and Thody, A.J. (1994) Alpha-Melanocyte Stimulating Hormone and Its Analogue $\mathrm{Nle}^{4} \mathrm{DPhe}{ }^{7}$ Alpha-MSH Affect Morphology, Tyrosinase Activity and Melanogenesis in Cultured Human Melanocytes. Journal of Cell Science, 107, 205-211.

[25] Lee, J., Jung, E., Park, J., Jung, K., Park, E., Kim, J., Hong, S., Park, J., Park, S., Lee, S. and Park, D. (2005) Glycyrrhizin Induces Melanogenesis by Elevating a cAMP Level in B16 Melanoma Cells. The Journal of Investigative Dermatology, 124, 405-411. http://dx.doi.org/10.1111/j.0022-202X.2004.23606.X

[26] Virador, V.M., Muller, J., Wu, X., Abdel-Malek, Z.A., Yu, Z.X., Ferrans, V.J., Kobayashi, N., Wakamatsu, K., Ito, S., Hammer, J.A. and Hearing, V.J. (2002) Influence of Alpha-Melanocyte-Stimulating Hormone and Ultraviolet Radiation on the Transfer of Melanosomes to Keratinocytes. FASEB Journal, 16, 105-107.

[27] King, R., Weilbaecher, K.N., McGill, G., Cooley, E., Mihm, M. and Fisher, D.E. (1999) Microphthalmia Transcription Factor. A Sensitive and Specific Melanocyte Marker for Melanoma Diagnosis. The American Journal of Pathology, 155, 731-738. http://dx.doi.org/10.1016/S0002-9440(10)65172-3

[28] Passeron, T., Bahadoran, P., Bertolotto, C., Chiaverini, C., Busca, R., Valony, G., Bille, K., Ortonne, J.P. and Ballotti, R. (2004) Cyclic AMP Promotes a Peripheral Distribution of Melanosomes and Stimulates Melanophilin/Slac2-a and Actin Association. FASEB Journal, 18, 989-991. http://dx.doi.org/10.1096/fj.03-1240fje

[29] Ramalho, J.S., Lopes, V.S., Tarafder, A.K., Seabra, M.C. and Hume, A.N. (2009) Myrip Uses Distinct Domains in the Cellular Activation of Myosin VA and Myosin VIIA in Melanosome Transport. Pigment Cell \& Melanoma Research, 22, 461-473. http://dx.doi.org/10.1111/j.1755-148X.2009.00567.X

[30] Lim, S.H., Kim, S.M., Lee, Y.W., Ahn, K.J. and Choe, Y.B. (2008) Change of Biophysical Properties of the Skin Caused by Ultraviolet Radiation-Induced Photodamage in Koreans. Skin Research and Technology, 14, 93-102.

[31] Masson, P.H. and Merot, F. (1992) Phototype and ITA Parameters for Determination of MED and SPF in Tanned and Untanned Subjects. Poster Preprints 17th IFSCC Congress, Yokohama, October 1992.

[32] Merot, F. and Masson, P.H. (1991) Phototype and Skin Color as Predictive Parameters for Determination of Minimal Erythemal Dose. Poster IFSCC Congress, Helsinki, September 1991. 